\title{
“El marro”, un juego tradicional y popular en la educación física española (1807-1936)
}

\section{"Prisoner's Bars", a traditional and popular game in the Spanish physical education (1807-1936)}

\author{
Jordi BRASÓ RIUS ${ }^{1}$ y Xavier TORREBADELLA FLIX ${ }^{2}$ \\ ${ }^{1}$ Instituto Centro de Alto Rendimiento Deportivo de Sant Cugat del Vallès \\ ${ }^{2}$ Universidad Autónoma de Barcelona
}

Recibido: Marzo 2014

Aceptado: Mayo 2014

\section{Resumen}

Desde tiempos históricamente remotos en Occidente, el juego del marro ha sido considerado por jóvenes y educadores un excelente medio de recreación y de endurecimiento físico. El rastreo de sus orígenes permite aproximarnos a una valoración pedagógica de esta práctica, entre 1807 y 1936 en España. El objetivo de estudio se centra en el análisis histórico y social del marro, hecho que permite obtener una aproximación cultural en el proceso de institucionalización de los juegos corporales en la educación física escolar contemporánea. Todo ello viene contextualizado por el proceso de civilización y de construcción social de un modelo burgués de valores. La metodología parte de una revisión documental de fuentes primarias y del análisis hermenéutico construido a partir de otros estudios historicistas relacionados. Los resultados obtenidos permiten referenciar el marro en España a partir del s. XVI. Se comprueba su protagonismo en el siglo XIX y los inicios del XX, jugado libremente por la juventud, pero también utilizado como actividad lúdico-pedagógica en las clases de educación física. Esta aparición en la educación normativizan su práctica, eliminando su carácter violento. Además, el juego fue un sustituto del fútbol cuando no se tenía balón, y era símbolo de regeneracionismo e identidad nacional. Juntamente con estas características, aparece su carácter disciplinado, su complejidad -mayor que numerosos deportes de equipo-, sus orígenes bélicos, su uso como actividad saludable, y su práctica mixta. De todo este análisis se concluye que el marro fue el primer juego de equipo de colaboración-oposición de carácter coeducativo. A la vez, se infiere que su práctica fue olvidada a causa de la importación de los deportes ingleses, especialmente el fútbol, mucho más normativizados. Su uso actual podría favorecer al trabajo competencial, además de ser beneficioso para trabajar las cualidades condicionales, cognitivas y la sociomotricidad.

Palabras clave: historia de la educación física, historia del deporte, juegos tradicionales, marro, regeneracionismo

\footnotetext{
Abstract

For a long time, the game of Prisoner's Bars or Prisoner's Base has been considered for youth and occidental educators an excellent way of recreation and physical hardening. The main 
objective of this article is the historical and social analysis of prisoner's base game. This analysis allows us to obtain a cultural approach of the institutionalization process of physical games in the contemporary school. The context of the study is the civilization and social construction of a bourgeois model of values. The methodology is based on a literature review of primary sources and the hermetic analysis built from other historicist related studies. The acquired results make reference to the Prisoner's game in Spain since the sixteenth century. It was confirmed its important role in the amount of games played by young people during the nineteenth and the beginning of twentieth century's. However, it was also used as a recreational-educational game during physical education classes. This use in education entails the apparition of rules avoiding the violent features of the game. Besides, this game was a substitute of football when there was no ball and it was a symbolism of regenerationism and national identity. In conjunction with these characteristics, it also appears its disciplined character, its complexity -higher than in numerous team sports-, its war origins, its use as healthy activity and its mixed gender practice. The main conclusion of this analysis is that Prisoner's game was the first collaboration-oppositional team game with coeducational use. In addition, it is inferred that its practice decreased due to the English sports importation, which has more detailed rules, especially soccer. Nowadays, the use of Prisoner's game could improve the competence work; besides it is beneficial to enhance additional abilities related with cognition and motor skills.

Keywords: history of physical education, history of sport, traditional games, prisoner's bars, prisoner's base, regenerationism

En estos últimos años el juego motor se ha presentado bajo el objeto de recuperar la raíz folklórica o la etnografía. Repertorios de juegos tradicionales o populares se han incorporado en la bibliografía de la educación física, no obstante solamente se limitan a señalar su descripción. Como cita González (1993), son pocos los trabajos que aportan una visión fenomenológica sobre los orígenes del juego deportivo. El estudio de los juegos corporales bajo una adscripción a la antropología histórica o cultural, puede ayudar a una hermenéutica sociocultural sobre los orígenes del deporte contemporáneo (Hernández, 2004). Sin embargo, pensamos que para alcanzar una interpretación ajustada sobre el origen de los juegos deportivos no existen teorías únicas. Las tesis interpretativas pueden superponerse unas a otras, fundiéndose y hasta confundiéndose en una multiplicidad social encadenada de intenciones diferentes, pero dependientes entre ellas y estructuradas en función de los matices de cada doctrina o posición de ideas que expresa un determinado grupo social y que las sustenta para lograr una finalidad concreta o particular, que incluso se transforma con el tiempo.

Generalizamos, por tanto, la aceptación de la tesis de Huizinga (1957), que sostiene que en la evolución antropológica del juego como elemento lúdico y festivo, se consolidaron los fundamentos sociales forjadores de la cultura moderna, la cual se manifiesta actualmente en el asentamiento de una larga tradición y supervivencia de muchos de los juegos corporales de antaño.

En España, las actividades físico recreativas de los siglos XVI y XVII han quedado testimoniadas en las obras de Cobarruvias (1543), Alcocer (1559), Caro (1978), Cobarruvias (1611) o Remón (1623). Asimismo varias investigaciones han constituido 
una contrastada base historicista con la que pueden iniciarse estudios más específicos (Álvarez, 1995; Bantulà, 2005, 2006, 2008; Bantulà y Vilanou, 2009; Hernández, 2004; Hesse, 1967; Mayor, 2002; Payà, 2006, 2007; Pelegrín, 1998, 2000).

El nuevo horizonte que actualmente se presenta con la utilización de las nuevas tecnologías y del acceso inmediato a las hemerotecas digitales, nos permite avanzar hacia el rescate de juegos perdidos y olvidados o, en todo caso, de algunos juegos que hasta no hace mucho estuvieron en la memoria de nuestros mayores. Uno de éstos es, el todavía conocido con el nombre de marro; una recreación física que está a punto de desaparecer por completo.

Así es, por tanto, que en la presente aportación abordamos como objeto de estudio el juego del marro en España en una interpretación histórica y social que se contextualiza especialmente en el ámbito del proceso de institucionalización de la educación física escolar entre 1807 y 1936.

La metodología se ha construido a partir de la localización bibliográfica de las obras de la época -fuentes primarias- facilitadas especialmente por los repertorios bibliográficos más recientes (Torrebadella, 2009, 2011), y completados con el soporte de la búsqueda en las hemerotecas digitales de la Biblioteca Nacional y de la Biblioteca de Cataluña (Arxiu de revistes catalanes antigues: ARCA). Asimismo, la revisión documental y el análisis de los datos obtenidos se han organizado cronológicamente en una fase hermenéutica y reforzada con estudios historicistas y fuentes secundarias.

Con este estudio aportamos datos y fuentes que pueden ayudar a sedimentar una historia social de la pedagogía de la educación física. Además, se pretende contribuir en la elaboración de una antropología histórica y cultural del deporte en España, que como citan Medina y Sánchez (2003) todavía no tiene una consolidada trayectoria ni institucionalización.

\section{El juego del marro: orígenes y noticias de su juego en torno a la educación física}

Como citaba Ricardo Rubio (1893), para poder presentar una historia de los juegos corporales en España deberíamos perdernos en la noche de los tiempos. Sin embargo, para rastrear el origen del juego del marro nos sirven las anotaciones de Pelegrín (1998), que descubre en el Diccionario de Autoridades la voz del juego, marcando diferentes acepciones de otros juegos que también adoptaron este nombre: "Marro. Se llama también el regate o hurto del cuerpo para no ser cogido y burlar al que persigue” y a otro juego de precisión que consiste en la suerte de acercar una piedra lanzada lo más cerca posible a un objeto clavado en el suelo (Autoridades, 1734, 504). En esta época, el juego del marro también se definió del siguiente modo:

Marro. Significa también juego parecido al que llaman Moros y Christianos, en que puestos dos bandos de una parte y otra encontrados, dexando suficiente campo en medio, salen de uno y otro a coger o tocar el contrario: y el arte consiste en huir el cuerpo el uno al otro, para que no le cojan o toquen, retirándose a su bando, 
porque si le tocan queda prisionero, por lo que le dieron este nombre (Autoridades, 1734, 504).

Pelegrín (1998) apunta que en los siglos XVI y XVII el término 'marro' fue asignado a otros juegos. El marro tiene mucha similitud con el "Juego de los cautivos" que Alonso Ledesma (1562-1623) incorporaba en 1605, en Juegos de Noche Buena Moralizados (Ledesma, 1611; Pelegrín, 1998, 2000; Sancha, 1855). Cristóbal Suárez $(1630,272)$ mencionaba que entre los juegos de su época eran conocidos "los ladrones, los cautivos, guardarropa, marro, velorto, chueca, \&”. Ledesma desplaza de los juegos el valor moralizante y ascético del barroco español, dominado por el poder católico que ejercía el Estado. El juego de "Los cautivos", se presenta como la terrenal lucha de los cristianos frente a la tentación del pecado (Sancha, 1855).

La popularidad del juego se comprueba por la presencia del vocablo en varios de los diccionarios de la época. Así detectamos la traducción francesa de marro, por jeu des barres (Cormon, 1776; Sejournant, 1759). Estos registros documentales nos llevan a sostener la suposición inicial, que el primitivo juego en España era referenciado hacia principios del siglo XVII con el nombre de Los Cautivos, y que hacia finales de este siglo y principios del XVIII, se conoció como Marro, tal y como recoge el Diccionario de Autoridades (1734).

Podemos sentenciar que el marro empezó a tomar carta de naturaleza en la educación física escolar española a partir de 1807 con la incorporación de $L a$ gimnástica o escuela de la juventud, obra de Johann Christoph Friedrich Guts Muths (1759-1839), cuya traducción española -de Mariano de Iborra- se tomó de la edición francesa de Amar y Jauffret. A partir de la publicación en 1793 de Gymnastik für die Jugend, la influencia de Guts Muths revalidó la percepción educativa de los juegos corporales.

La versión española de La gimnástica o escuela de la juventud, dedicada al director del Real Seminario de Nobles, indica la certeza que en dicha institución se practicara el juego del marro. Sin embargo, en esta obra el juego adoptaba el nombre francés Las barras -Les Barres- indicando que era "conocido en España con el nombre de moros y cristianos” (Amar y Jauffret, 1807, 121 y ss.). Sobre su descripción destacamos la elocuente introducción cuando se cita la importancia que tenía el juego en Atenas, indicando, como también sucedía en la edición francesa que "este juego en todos los países, menos en el nuestro es exercicio de las jóvenes” (p. 122). Subrayamos este rasgo porque hace del juego el primer contenido coeducativo de la educación física, hecho que muestra la clara influencia de Platón, el cual aconsejaba que los juegos y retos de carreras fuesen practicados tanto por hombres como por mujeres (Mercurial, 1845). En España esta obra fue recomendada por sus excelentes valores educativos (J. L. N. P. R., 1808) y la Gaceta de Madrid -10/11/1807- la presentó como una "obra recomendable para los colegios, seminarios y demás casas de educación” (Pelegrín, 2000, 44).

A partir del siglo XIX, los juegos recreativos de los jóvenes y, en especial los juegos corporales, se enrolan en un proceso de pedagogización e institucionalización escolar. Estos juegos siguen configurando el proceso de sociabilización o civilización 
de los antiguos juegos corporales, que según Elias y Dunning (1992) son reglados y convertidos, algunos de ellos, en las prácticas deportivas contemporáneas.

Asimismo, las intervenciones pedagógicas de Salzmann, Guts-Muts, Pestalozzi, Clias o Amorós (Diem, 1966), sirven como modelo para incorporar los ejercicios o juegos gimnásticos y esbozar proyectos educativos. Los juegos corporales son presentados como excelentes medios para organizar la educación física escolar pero, además, cumplen una función preventiva y reparadora ante la corrupción higiénica y moral que acostumbraba a presentarse en los colegios internados (Vázquez y Seoane, 2004). Como en Francia, y por la influencia pedagógica que se recibió de este país, podemos suponer que también en los colegios internados de España el juego de barres -el marro- era el preferido para la recreación, la instrucción física y la formación del carácter del alumnado (Pavet, 1827; Simon, 1827).

En el Manual de higiene (1831) del médico Ignacio Pusalgas (1790-1874), el capítulo dedicado al ejercicio, aún hoy, y salvando el paso del tiempo, tendría una cierta consideración. En él se tratan los diferentes géneros de ejercicio ya fuera como recreación de la infancia o de la juventud, como parte esencial de la educación en el desarrollo corporal o como prescripción higiénico-médica para conservar o restablecer la salud. Pusalgas ubicó los juegos corporales de la infancia como uno de los medios higiénicos mejor probados para asegurar el desarrollo corporal y como una parte esencial en la educación, como así se hacía en los colegios anglosajones. Hacía un repaso de los principales juegos destacando sus cualidades higiénicas. Además, el ejercicio considerado como parte esencial de la educación era abordado a través de la aplicación práctica que tuvieron los clásicos sobre la gimnástica. Así presentaba la conveniencia de establecer la gimnástica en los colegios, como antiguamente en Grecia: " $1^{\circ}$ para endurecer y fortificar los jóvenes destinados a las fatigas de la guerra, $2^{\circ}$ para conservar la salud y restablecerla, $3^{\circ}$ para formar los atletas, dotados de las más grandes fuerzas de que es susceptible el cuerpo humano” (Pusalgas, 1831, 76). Pusalgas destacó los juegos corporales de carrera, que ya introducían las escuelas de diferentes países y la mayoría de los colegios de Inglaterra como así sucedía con "el juego del marro (parecido al que llaman moros y cristianos) o de los prisioneros" (p. 74).

Podemos comprobar que antes de llegar a la primera mitad del siglo XIX, el marro ya tenía una cierta popularidad, puesto que la improvisación del juego en las zonas urbanas provocaba frecuentes altercados a los transeúntes. En 1835 en las Ordenanzas municipales de Tudela, el Art. 372 exponía que: "No se podrá jugar al marro en las calles, plazas, ni paseos públicos, ni a pelota en la pared que se halle situada en paraje que se interrumpa el libre tránsito a las gentes, bajo la pena de una peseta, a que serán responsables los padres o tutores por sus hijos menores” (Tudela, 1835, 159).

El tratado de Marco Antonio Jullien (1845), Ensayo General de educación física, moral e intelectual, consideró el juego del marro como un excelente medio para contribuir a la educación física a partir de los ocho años:

Algunos juegos de la guerra proporcionan a los niños mil ocasiones de desarrollar su velocidad persiguiendo al enemigo su agudeza para escapar á sus 
emboscadas su emulación para hacer triunfar el partido a que pertenecen; su elocuencia y energía para excitar á sus jóvenes compañeros su valor generosidad para si están en peligro en fin justicia y amor á la virtud decidir en los casos dudosos De este modo la perfección física está en relación con los otros dos ramos de la educación. El cuerpo se desarrolla como el entendimiento y el carácter La vivacidad la libertad la alegría que todos sus juegos favorecen circulación de la sangre y los humores el apetito el sueño el aumento de las fuerzas la salud la alteración del temperamento y las enfermedades demasiado comunes en niños sedentarios y esclavos é inmovibles y desgraciados que son por regular groseros macilentos valetudinarios y el vigor del cuerpo y la salud se hallan enlazados con satisfacción del alma con igualdad de carácter con gusto el buen humor y alegría. (Jullien, 1845, 260-262).

Como trata Torrebadella (2013), la obra de Jullien representó la influencia recuperada del modelo pedagógico de Pestalozzi y del sistema de educación física del compatriota exiliado en Francia, Francisco Amorós.

José Simón (1992) destaca que los alumnos del Instituto de San Isidro de Madrid solían frecuentar el llamado "Campo del Moro" -alrededores del Palacio Real- para disputar partidas de marro. Uno de estos alumnos era el literato José Fernández Bremón (1902, 266), que recordaba como hacia mitad del siglo XIX con todos sus compañeros del Instituto se desplazaban en tropel "al Campo del Moro para jugar un marro". Por lo que se desprende, parece que este lugar fue durante muchos años una concurrida zona de recreación, en la que también solían organizar partidas de marro los alumnos universitarios (Fernández, 1898).

El juego del marro fue apareciendo a lo largo de todo el siglo XIX, aunque según los autores recibió nombres diversos (Pelegrín, 1998). Así R. C. $(1847,51)$ le llama “Combate de carreras”. Esta identificación guerrera también vendría refrendada en las declaraciones de otros autores:

En nuestros días no se representa este ejercicio más que por el juego del marro que era antiguamente un combate de hombres en un espacio cerrado de barreras. Esta manera de correr es de las más saludables, y por eso debe recomendarse mucho: no solo tiene la ventaja de desarrollar las fuerzas y de dar agilidad, sino también la de estimular el valor y la destreza. Ofrece la imagen de un combate, pero de un combate inofensivo; la inteligencia del Gefe tiene tanta parte en la victoria como el valor de los soldados: necesita conocimientos estratégicos, elije el momento de ataque, calcula los accidentes del terreno, reconoce la parte débil del enemigo y mide con una ojeada los círculos y vueltas que es necesario dar para sorprender; y cuando por medio de un golpe atrevido ha librado sus prisioneros, $o$ tomando el campo contrario, queda cubierto de gloria. (Mutel, 1846, 152)

Basilio Sebastián Castellanos (1849) refiriéndose al juego de bandos o al conocido como Daca de la china, considerados por Pelegrín (1998) como sucedáneos al juego del marro, decía que era muy común "ver á nuestros muchachos del pueblo entretenerse en juegos de ejercicio, que, por su carácter, parecen simulacros de la guerra” (Castellanos, 1849, 296). 
Fernández de los Ríos (1852) aconsejaba, del mismo modo que ya hicieran Amar y Jauffret (1807) el juego a las señoritas expresándose del siguiente modo:

En nuestro sentir vale más esta ocupación que la lectura inspirada y con mucha frecuencia peligrosa de una mala novela, o la murmuración más insípida todavía, de la mayor parte de las reuniones o eterna monotonía de todos esos pretendidos juegos de habilidad que tan poco discurso suponen en los que se divierten en ellos. Jugado por jóvenes de los dos sexos este juego es todavía más bello porque mil accidentes graciosos aumentan su atractivo. (Fernández, 1852, 20).

En 1803 el DRAE varía la identificación del juego respecto al Diccionario de Autoridades (1734): “Juego en que puestos dos bandos de una y otra parte encontrados, dexando suficiente campo en medio, salen de uno y otro hasta la mitad de él a coger, o tocar el contrario, y el arte consiste en huir el cuerpo el uno al otro, para que no le cojan o toquen, retirándose a su bando" (R. A. E., 1803, 541). Más tarde, el DRAE dice que "este juego se conoce con otros varios nombres" (R. A. E., 1852, 442).

El marro aparecerá frecuentemente en algunos tratados de juegos (Fernández, 1852; Fernández, 1864; Hernández, ca. 1900; López, 1855; Marco y Ochoa, 1896; Maspons, 1874; Un aficionado, ca. 1905; Un papa, 1897) y también en manuales o programas de educación física escolar de finales del siglo XIX (Ferrer, 1893; García-Fraguas, 1896, 1897; Macorra, 1894; Sanz, 1894). Así es que a finales del siglo XIX, los juegos tradicionales y los autóctonos españoles, como el marro, se hacen muy recomendables (Payà, 2006).

El doctor Fonssagrives (1877) en La Instrucción Pública, al ocuparse de la educación física de las niñas y de los juegos más convenientes para su desarrollo, destacaba que aún y las características viriles que contiene el juego del marro, por su aspecto guerrero, las niñas no desechaban demostrar la rapidez de su carrera.

Eduardo de Palacio $(1882,158)$ revelaba que viendo jugar a los niños se pueden descubrir sus inclinaciones y a la tarea que acaso se dedicaran al llegar a mayores. Así el jugar "al marro indica ciertas aficiones políticas entre los tiernos infantes; van á ver quién puede á quién, y cuál es el bando que triunfa”.

Marco y Ochoa $(1896,839)$ citaban que se trataba de una de las "diversiones favoritas de la juventud, sobre todo en los grandes colegios”. Hernández (ca. 1900), mencionaba que el juego del marro es el origen de otros juegos. Para Méndez (1907, 190) el marro "es un juego infantil de los dignos, en el cual se ejercitan los músculos, porque se corre incesantemente, y por el cual se adquieren ciertos hábitos de amor propio". Fueron muchas las voces pedagógicas que aceptaron este juego para la educación física. Del marro se decía que era entre todos los juegos el que más se parecía a la guerra y, también el más preferido por los estudiantes (Castellanos, 1849; Fonssagrives, 1877; Mancuvrier, 1890; Marco y Ochoa, 1896).

Hacia finales del ochocientos, el abuso de la gimnástica fue criticado por ambientes pedagógicos de la Escuela Nueva. Pedro de Alcántara García (1882) -primer pedagogo contemporáneo español que presentó una amplia exposición de la teoría del juego en el ámbito de la educación física-, en alusión al momento histórico de finales del siglo 
XIX conocido como renacimiento físico, expuso las razones de porque los juegos corporales estaban desplazando a los ejercicios gimnásticos de los programas escolares. La pedagogía moderna tomaba como preferencia los juegos corporales, porque además de cumplir con todas las ventajas higiénicas de la gimnástica proporcionaban una mayor sociabilización, recreación y desarrollo personal.

Alcántara García citaba que de todos los juegos de carreras, el del marro tenía su preferencia entre los padres:

[...] -en francés jeu de barres: es parecido al que llaman moros y cristianos-, que pertenece, sin duda, más especialmente al sexo masculino por sus pasos guerreros, pero en el que las niñas no se desdeñan menos, nuevas Atlantes, de ensayar con rapidez la carrera. Conviene a la solicitud de las madres no dejar que este juego se prolongue hasta un límite tal que el cuerpo se empape de sudor y el corazón lata con impetuosidad. (García, 1882, 132).

Como ha señalado Payà (2006, 333), la maestra valenciana de enseñanza superior, Natalia Castro (1895a, 1895b) argumentó, en la Asamblea del magisterio de Sevilla, Valencia y Barcelona, que de todos los juegos susceptibles que disponen las niñas para ejercitarse es, "especialmente en el archiconocido juego español del marro", el que se puede presentar en las escuelas como uno de los mejores medios para incidir en la formación física y del carácter. Recordemos que así ya lo había indicado el Dr. Fonssagrives (1877), autor que Pedro de Alcántara García reconoció como la primera referencia pedagógica para organizar la educación física de las niñas.

En los colegios de la Institución Libre de Enseñanza (ILE), Francisco Giner, influenciado notablemente por el valor educativo del deporte inglés, fue uno de los máximos defensores en la introducción del sistema de educación física de los colegios ingleses (López, 1998a, 1998b; Payà, 2004). En 1887, Giner aprovechó una solicitud al Ayuntamiento de Madrid para reclamar la habilitación de campos de juego apropiados para los niños y dejó expuesto que desde 1878:

[...] nuestros discípulos, cuyo ejemplo no ha sido inútil para otras corporaciones y establecimientos, han salido diariamente con sus profesores ( nada de inspectores ni ayudantes!) entre las clases de la mañana y las de la tarde, no ha paseo, sino a jugar en algún sitio abierto, además de consagrar por entero toda la tarde del miércoles, y con frecuencia las mañanas de los domingos, a partidas organizadas de pelota, marro, rounders, liebres [...] (Giner, 1887, 338)

El marro era uno de los preferidos por la escuela madrileña de la ILE y Ricardo Rubio (1889) lo tenía como uno de "juegos propios de nuestro país”, del que dice ser:

[...] el gran juego de lucha y esfuerzo, en que cada jugador no puede contar sino consigo mismo, y tiene que medir sus fuerzas para saber hasta dónde puede arriesgarse, y ha de medir los contrarios que pueden salir en contra suya; en el que hay la carrera violenta, el regate, el esfuerzo para desasirse y el empuje para liberar a los compañeros. (Rubio, 1889, 51). 
El doctor Alejandro San Martín (1889) precisaba que el modelo pedagógico de Pierre de Coubertin en España estaba representado desde hacía ya una década por la ILE. Al respecto, argumentó la necesidad de superar la rivalidad intrínseca entre partidarios de la gimnástica y el sport inglés mediante la recuperación de los juegos corporales, cuya implantación en España era de extrema necesidad. San Martín (1889), director de la Escuela Central de Gimnástica (1887-1892), ocupándose de la campaña iniciada por Coubertin y Ph. Daryl -pseudónimo de Pascal Grousset-, para propagar el modelo de educación física inglés, puso objeciones pedagógicas y morales a la orientación militarista con la que se dirigían y se fomentaban los juegos corporales en la República francesa.

El juego del marro fue defendido pedagógicamente en Francia por el profesor Fernando Lagrange. Pero como citaba Mancuvrier (1890), del mismo modo que en España, el juego tenía el peligro, injustamente, de caer en el olvido a causa de la importación de otros juegos extranjeros.

La extensión geográfica que alcanzó la popularidad del marro quedaba expuesta en el Romance que de este juego presentó Manuel Ossorio (1891, 89-92). Como subrayó Guimerá (1889, 36), en el BILE, “en los juegos ven los ingleses, no tan sólo el ejercicio físico sino un embrión de vida social en la emulación digna, la disciplina, la sumisión al mando y a la autoridad, la lealtad y la abnegación que tienen en efecto los juegos”. Pero para Guimerá $(1889,36)$ los juegos corporales fuertes al aire libre -como el del marro- también poseían una ventajosa influencia moralizadora, porque actuaban como calmante en los sentidos y en la imaginación de los jóvenes que "desean descansar y dormir y van a la cama soñando en los juegos del siguiente día lo cual constituye un poderosa salvaguarda contra la corrupción impidiendo unas veces que penetre y otras que extienda, cuando accidentalmente ha penetrado". Es decir, que el juego se edificaba pedagógicamente como uno de los mejores preventivos ante la contagio de la masturbación entre los jóvenes (Vázquez y Seoane, 2004).

El juego del marro también obedecía a esta sociabilización de la conducta moral, como así lo manifestaba Ricardo Rubio (1893) al argumentar que los juegos corporales al aire libre en la edad escolar cumplían una función higiénico-pedagógica. En la ILE los juegos tradicionales al aire libre fueron presentados como un poderoso estímulo de la ejercitación física y desarrollo del carácter. Rubio matizaba que cada uno de los juegos que practicaba la ILE aportaba un valor educativo. Del marro citaba:

[...] exige de los directores un ejercicio del principio de autoridad, de las condiciones de mando en todos los momentos, así como el arrojo y la resistencia en los casos apurados, y de los demás jugadores la subordinación siempre, la protección del perseguido en muchas ocasiones, y la gran idea de la responsabilidad en los que custodian los 'parados'; citando muy a la ligera y solo los más importantes. (Rubio, 1893, 149)

Alcántara García (1896) aconsejaba atender los juegos corporales con aquellos que más agradaban a los niños. Generalmente éstos eran los que solían participar entre dos bandos que luchaban en un partido y que más y mejor ejercitaban las fuerzas físicas y 
la destreza. Entre estos juegos, el del marro era el más idóneo a las citadas condiciones y, sobre él Alcántara García aclaraba que:

[...] no el que consiste en tirar a un bolo con el marrón, sino el que, colocados los jugadores en dos bandos, uno en frente de otro, dejando suficiente campo en medio, sale cada individuo hasta la mitad de él a coger a su contrario, consistiendo el arte en huir el cuerpo, no dejándose coger ni tocar, y retirándose a su bando. (García, 1896, 422)

El profesor Ph. Tissié (1899, 361) aconseja la utilidad del juego del marro como ejercicio deportivo de persecución reglamentada para los niños de edad mediana y, los deportes del fútbol y el rugby, de los que decía "que son como un marro simplificado", para los alumnos mayores.

Guibert $(1990,31)$ apunta que el marro era uno de los preferidos de los alumnos y de los maestros, que solía realizarse a propósito de recrear y ejercitar al alumnado en los paseos escolares: "Muy recomendado, así como todos aquellos que intervienen partidos. Son muy bien acogidos por los niños dada la destreza que necesitan en su ejecución y el atractivo que origina, entre ellos, la lucha y la competición”. El mismo Padre Manjón prefería los juegos y los ejercicios naturales al aire libre, antes que la gimnástica de aparatos. Por ello pedía campos escolares para practicar juegos como el del marro que, entre otros, contribuía al alejamiento de los jóvenes del sedentarismo, de los vicios y prestaba servició a "la regeneración de la raza degenerada" (Gálvez, 1940, 360-361).

A partir de la crisis de 1898, la educación física absorbió y se enfatizó, todavía más, en el discurso regeneracionista de la época, que ya venía manifestándose en el ámbito profesional de los llamados apóstoles de la educación física, desde hacía varias décadas (Torrebadella, 2012a). Profesores como Marcelo Sanz Romo (1859-1942), en Madrid, o David Ferrer Mitayna (1848-1901), en Barcelona, se erigieron como líderes indiscutibles y reconocidos por todo el colectivo profesional. El profesor Sanz desde las páginas de la Educación Física Nacional, portavoz de la Asociación de Profesores Oficiales de Gimnástica y de la Liga Madrileña de Educación física, presentó una propaganda a favor de los juegos tradicionales españoles como reacción a la invasión de los deportes de origen anglosajón. Así publicó varios opúsculos en los que expuso las reglas de los juegos tradicionales de mayor popularidad e identidad nacional (Marín, 2009). En uno de estos folletos se citaba:

Todos los juegos, después de adaptarlos a las distintas edades después de expurgarlos de todas aquellas causas que pudieran hacer peligrosos bajo el punto de vista físico y moral, irán apareciendo en folletos como el presente.

Los distintos juegos que en cada región de España se practican, los daremos a conocer; para ello tenemos, como queda dicho, personas conocedoras de las costumbres en distintas regiones.

En cuanto a los juegos de otros países, también serán objeto de nuestra intención; pero en el extranjero tienen ya hecho el trabajo que nosotros vamos a hacer con los nacionales. (Barrilero, 1900, 4) 
David Ferrer (1900b), profesor de educación física del Instituto de Barcelona, inició una amplia campaña en favor de los juegos corporales y de los campos de juego públicos. En la Cartilla gimnástica higiénica, Ferrer (1901, 41-42) incorporó la descripción del juego del marro y que anteriormente ya había divulgado en Los Deportes (Cartilla de gimnasia higiénica, 1900a). Así es como el juego se agregó también en los concursos gimnásticos deportivos que organizó la Federación Gimnástica Española (1902) en Barcelona.

El profesor Daviu $(1902,41)$ desde el Magisterio Balear afirmaba que el marro era "una de las diversiones favoritas de la juventud española, sobre todo en los colegios numerosos", y sostenía que era "uno de los más animados sin juguetes, y sin duda el más difícil y que más gusta a los niños”. Sin embargo, indicaba que por el gran espacio que requiere el juego solamente tenía lugar en las excursiones y en los paseos escolares. Daviu concretó que en el juego existen muchas variantes. Entre las más importantes, destacaba la de hacer prisioneros solamente tocando al adversario, y no agarrando como habitualmente sucedía. Sobre las consecuencias desagradables del juego, aparte de las continuas riñas, también se encontraba la prohibición de los padres o de los colegios porque solían romperse la ropa al agarrarse (Rumrep, 1903).

Estas argumentaciones son prueba suficiente del valor pedagógico que se le confirió al juego del marro en el magisterio español y, sobre todo, en la ILE, unas décadas antes que quedara casi definitivamente desplazado por la sencillez y estimulante acogida del fútbol (Torrebadella, 2012b). No obstante, el juego continuó prevaleciendo entre los jóvenes, y servía de estímulo recreativo ante la ausencia del entonces apreciado y costoso balón de fútbol.

La Compañía Madrileña de Urbanización, encargada de consagrar el proyecto urbanístico de la Ciudad Lineal en Madrid diseñado por Arturo Soria Mata (18441820), desarrolló a principios del siglo XX un extenso programa de cultura física contextualizado en el pensamiento regeneracionista. Bajo la influencia del deporte anglosajón se comprometía a dignificar los campeonatos deportivos entre los escolares y a incorporar "entre los deportes gimnásticos tres verdaderamente clásicos, genuinamente españoles: el juego del marro, el juego del paso y el tiro de la barra” (Higiene, 1905, 3).

En el Manual de juegos y deportes publicado en la Biblioteca Popular de Saturnino Calleja, el marro se presentaba indicando que "en algunas partes de España llaman también a este juego Rescate, nombre que conviene mejor a la índole de la partida” (Un aficionado, ca. 1905, 91).

En los sucesivos años, los juegos corporales tomarán mayor importancia en los manuales de texto de las escuelas normales y en algunas de las guías docentes para la educación física de primera enseñanza, que no evitan la incorporación del juego del marro o su alusión (Escribano, 1912; Nociones de ejercicios corporales, 1909). Tanta era su popularidad que también llegaba al Reglamento Provisional de Gimnasia para Infantería de 1911. En él se introducía la gimnasia sueca como el nuevo método de educación física, en la formación del soldado pero también un el capítulo VI dedicado a los "Juegos deportivos" mostraban, como ejemplos, aquellos juegos tradicionales, 
que mejor aplicación física tenían, para la preparación del soldado destacándose el del marro o rescate (Ministerio de la Guerra, 1911).

Podemos apreciar, pues, que el juego del marro fue incorporado en los programas de educación física de numerosas escuelas e institutos. Así sucedía en el Instituto de Palma de Mallorca con el profesor Eusebio Ferrer (1919). En los años veinte, cuando el fútbol adquiría notoria popularidad entre las clases medias, el juego del marro se incorporaba todavía en los manuales o tratados de educación física escolar (Casals, ca. 1930; Condo, 1927; Eleizegui, 1924; Hernández y Caballero, ca. 1928).

Todo y el aumento que ejercía el protagonismo del deporte en la escena escolar del primer tercio del siglo $\mathrm{XX}$, surgieron modelos educativos que mantuvieron las raíces de los llamados juegos nacionales. Así se destacó el juego en la Escuela del Mar (19221938) de Pere Vergés en Barcelona, cuya presencia se distinguió como una poderosa herramienta de educación, y de tal modo formaba parte de la vida social de la escuela. La práctica del marro era regular y organizada, con entrenamientos, equipos, campeonatos, árbitros y un consejo, todo esto con los mismos alumnos como protagonistas (Anónimo, 1934; Borja, 1982).

El juego del marro continuó siendo muy popular durante los años treinta, sobre todo cuando no había balón con el que jugar, aspecto que sucedía muy a menudo. En esta época corrió la noticia que el Sr. Cervera capitán de un buque de la Armada Española embarrancó porque se retrasó jugando al marro, y no tuvo en cuenta el descenso de la marea (Temas navales..., 1932). Las anécdotas ocasionadas por el juego serían innumerables en la memoria de aquellos jóvenes. Por ejemplo, las podemos encontrar en la Guerra Civil, cuando el cese de las bombas permitía momentos de tranquilidad y asueto a los escolares (Farré, 2009). Otra anécdota en plena Guerra Civil proviene del cuento "Patio de Armas" (1961), de Ignacio Aldecoa, cuyo relato se inicia con la escena en el patio de un colegio:

¡Le jeu aux barres est plutôt un jeu français. Nos écoliers y jouent rarement. Voici ti quoi consiste ce jeu: les jouers, divisés en deux camps qui comptent un nombre égal de combattants, se rangent en ligne aux deux extrémités de l'emplacement choisi. Ils s'elancent de chaque camp et ils courent a la rencontre l'un de l'autre. Le joueur qui est touché avant de rentrer dans son camp est pris. Les prisonniers sont mis ti part; on peut essayer de les délivrer. La partie prend fin par la défaite ou simplement l'infériorité reconnue de l'un des deux camps." (Quevedo, 1992, 168)

Como aprecia Quevedo (1992, 169), este inicio sitúa un relato aterrador de la vida en donde el juego es convertido en realidad: "Aldecoa desde el principio nos da la clave del cuento; selecciona el mundo de los niños, sus espacios, sus juegos, para recrear y potenciar aún más toda la sinrazón de la guerra”. En este fragmento del relato aparecen términos que no escapan del imaginario colectivo de muchos de los juegos de la infancia: “combatientes”, “dos campos”, "prisioneros”, “derrota”, "inferioridad” y otros muchos tantos que podríamos añadir y que se revelan de las prácticas combativas como "capitanes”, “cazado”, "muerto” y “jefe”, términos que denotan claramente una acción beligerante. 
En la actualidad este juego es tratado todavía en la educación física como un juego tradicional o popular. Además, en cuanto al propio carácter motriz, como citan Lagardera y Lavega (2003), y como ya afirmaba Ph. Tissié (1899), el juego del marro tiene un alto grado de complejidad sociomotriz (Guillemard, Marchal, Parent, Parlebas, y Schmitt, 1988). Esta complejidad se refleja en la variedad de estrategias que pueden adoptarse y en el grado de disciplina que exige el juego. En palabras de Borja (1982, 109) "exige una disciplina y sumisión a uno que hace de jefe, si se quiere llevar ventaja en el juego" y también en las grandes dificultades en arbitrar los partidos (Bartrés, 1934).

La complejidad del juego del marro tiene su análisis en la definición de la lógica interna que lo sitúa entre los deportes colectivos de mayor inteligencia sociomotriz y, como trata Parlebas (2001, 449): "Un chico de ocho años puede jugar al fútbol correctamente, pero le faltará aptitud y eficacia para el juego del Marro”.

En Europa la longeva tradición del juego del marro perdió paulatinamente popularidad con la aparición de la institucionalización del deporte. Así en Inglaterra, Tom Brown, el célebre muchacho de la escuela de Rugby, jugaba al Prisioners' Base (Hughes, 1923) y en Francia Jacques Vingtras, el protagonista de L'enfant (1879), de la popular obra de Julles Vallès (1889) también jugaba a barres.

Pero el juego perdió protagonismo como así evocó Thomas Hardy (1840-1928), en la famosa novela victoriana Lejos del mundanal ruido (1874), que tanto éxito ha gozado hasta nuestros días. El autor incorporó en el prólogo, de la nueva edición de 1912, un pasaje en el que recordaba el juego de Prisioners' Base: “El juego del rescate que hace no mucho tiempo parecía gozar de una vitalidad eterna, a diferencia de otros juegos ya decaídos en desuso, es hoy por lo que he podido comprobar, absolutamente desconocido para las nuevas generaciones infantiles” (Hardy, 2011, 3). A poco nos equivocaríamos en intuir que una de las principales razones del olvido del juego debería ser vinculada a la omnipresencia que ejerció la foot-ball association y el football rugby y otros deportes en la sociedad escolar anglosajona. Esta situación, por extensión, también debería ser considerada en España por la pronta influencia que ejerció el proceso de deportivización de la juventud, y en especial el fútbol (Torrebadella, 2012b).

\section{Hacia una posible aplicación didáctica del marro}

La presencia singular de los juegos tradicionales en el currículo educativo actual es la mayor garantía de la recuperación del patrimonio pedagógico y de la conservación de la memoria educativa. Los juegos corporales del pasado contienen un extraordinario caudal antropológico y etnográfico, pues en ellos se deposita el legado histórico y los cimientos y valores de la sociedad contemporánea (Lavega, 2006). La asignatura de educación física puede y debe contribuir a recomponer el significado instructivo y simbólico de los juegos tradicionales de la motricidad. De este modo, reconocer en estos juegos el devenir histórico, las transformaciones y los pilares sociales forjadores de las sociedades o diversidades culturales, también forma parte de las propuestas docentes. Un enfoque constructivista puede acercar éstos juegos tradicionales para que 
el alumnado sea aprehensivo con las diferentes realidades de su entorno social. Esta ha sido una visión que tradicionalmente en la pedagogía escolar se ha venido manteniendo desde hace varios siglos. No es pues baladí, el sostener que juegos como el del marro aún tienen cabida en el currículo escolar de la educación física (Navarro \& Trigueros, 2008).

Todo este protagonismo del juego analizado, desde inicios del s. XIX hasta el primer tercio del s. XX, tendría que servir para reflexionar sobre la posibilidad de recuperarlo, específicamente en el ámbito educativo. Con el objetivo de facilitar el uso del juego del marro en el marco escolar, se sugieren algunas premisas didácticas.

Primeramente el juego podría presentarse a través de alguna lectura literaria sugestiva -fragmento de texto- en donde apareciese el marro. Este punto inicial daría cabida a la formulación de preguntas como: ¿sabéis que el marro fue uno de los juegos más populares que jamás ha existido?, ¿sabéis que casi siempre había disputas o riñas que tenían que resolverse en el consenso de los participantes?, ¿`sabéis que quiere decir: aquí hay marro? ¿queréis saber cómo se juega?, ¿deseáis jugar?, ¿tal vez podríamos encontrar en algún sitio la descripción del juego?, ¿Podemos preguntar a la gente mayor?, etc. Estas y otras preguntas parecidas pueden despertar el interés del alumnado y aproximarle a un proyecto de grupo con el objeto de participar en una "arqueología" del juego.

La contextualización del grupo se fijaría a partir del tercer ciclo de educación Primaria. En atención al marco curricular de las competencias básicas, el marro puede ser presentado desde el área de Educación Física como un proyecto interdisciplinar hacia la cesión de la autonomía (Becerro-Mina y Torrebadella-Flix, 2013). Esta cuestión se referiría a tratar el juego desde diferentes visiones, como por ejemplo:

- tratando la competencia motriz, mediante la propia práctica del marro y de sus juegos parecidos;

- trabajando el uso de la lectura, la recitación y la comprensión, mediante textos como los Juegos de los niños en las escuelas y colegios (Hernández, ca. 1900), o el romance de "El marro" de Ossorio (1891);

- analizando la perduración del juego en la literatura universal -como en las obras, de acceso abierto, de Hardy, Vallès o Aldecoa-;

- reflexionando sobre los valores sociales inherentes en el juego y relacionándolos con los actuales;

- proponiendo una normativa del juego, adaptándolo al momento actual, y buscando nuevas variantes;

- organizando un campeonato, con todos los aspectos de gestión que son requeridos para su puesta en marcha;

- tratándolo como objeto de análisis estadístico, mediante el uso de encuestas a familiares, conocidos o gente mayor, y valorando su perdurabilidad por zonas de procedencia;

- analizando las diferencias de nombre y de práctica del juego en diferentes países, hecho que implicaría el uso de las lenguas extranjeras. 
Es evidente que el marro, como otros tantos juegos tradicionales, podría tener cabida en la actualidad (Devis, 2008). El juego responde a numerosos objetivos y contenidos curriculares de la educación física, además de poder utilizarse como un elemento de relación con otras materias y, por tanto, favoreciendo al trabajo competencial. Como juego colectivo de colaboración-oposición, la diferenciación de roles, la dualidad y el simbolismo, las organizaciones y relaciones de la estrategia, la tomas de decisiones y la resolución permanente de problemas, la gestión del riesgo, la autopercepción de la corporalidad o la coeducación, son condiciones que forman parte también de un desarrollo corpóreo amplio.

El juego tradicional como práctica libre y organizada por el alumnado tiene en el modelo pedagógico de Pere Vergés -aplicado en la antigua Escuela del Mar y en las colonias de Vilamar-, esta misma línea constructivista (Brasó y Torrebadella, 2014). Hoy, el currículo en torno a las competencias, garantiza la posibilidad de actuar, con esta misma dimensión integradora y cognoscitiva en la adquisición y transferencia del saber y del ser.

La manera de organizar el juego del marro en la antigua Escuela del Mar, es quizá la propuesta que habría que tener más en cuenta, ya que es de fácil adaptabilidad al momento actual, puesto que además de tratar metodológicamente el juego como un proyecto en sí, no queda despojado de la responsabilidad docente en la educación de los seres humanos.

\section{A modo de conclusión}

Revolviendo el desván de los juegos tradicionales hemos rescatado del olvido uno de los juegos perdidos. El marro fue tenido como un juego o deporte genuinamente nacional, pero sus ancestros nos remontan seguramente hasta la Grecia antigua, que lo concibieron como un juego simplificado o modificado de preparación para la guerra (Andreu, 2009). Podríamos llegar a pensar que el juego del marro se fue incorporando en España por influencia francesa hacia el siglo XVI, pero también pudiera ser que ya existiera desde mucho antes.

En España el juego del marro encarnó el deporte de equipo -sin balón- más representativo y popular de todo el siglo XIX y de la primera mitad del siglo XX. Fue un juego de patio, pero también un juego marcado por un interés pedagógico y llevado a la educación física. Principalmente fue concebido como un juego de estímulo para los niños y fue presentado como preparación premilitar, aunque no se negó la participación al sexo femenino. Podemos llegar a sostener que se presentó como el primer juego de equipo de colaboración-oposición de carácter (co)educativo.

Especialmente, el juego del marro contribuye, en plena expansión industrial y burguesa, a fijarse entre todo el elenco de los juegos corporales como el juego preferido para la educación física escolar. Los tratados de la época normativizan el juego y despojan de él algunas de sus características o variantes violentas, con el propósito de garantizar el proceso de civilización y de construcción social del modelo de valores burgués. Así el marro fue un juego moralmente aceptado, aparte de ser 
considerado como un antídoto al sedentarismo de los muchachos. Además tenía como ingredientes el mantener las virtudes sociales exigidas: la disciplina de las órdenes del jefe; la astucia que requería la condición de ser un juego estratégico; la toma de decisiones arriesgadas; el heroísmo en la protección de los compañeros; el celo en la vigilancia de los presos, y la agilidad y la resistencia de un juego atlético y viril. Por estos motivos, la práctica tuvo tanto éxito en el ámbito educativo y recreativo de las escuelas. Fue en este caso, ya entonces, el juego del marro el dispositivo tecnológico de una tradición impuesta por la cultura dominante en la educación física. Hoy esta intervención socializadora tiene al deporte que se filtra como colonizador de la educación física escolar (Vicente y Brozas, 1996).

Además, el marro fue un substituto del fútbol cuando se carecía de balón. Sin embargo, en España el marro se presentó como un símbolo de regeneracionismo y de identidad nacional: el deporte español frente al sport extranjero, aunque el fútbol le ganó socialmente la partida.

Como citaba Kant (2003), los juegos de los niños son muy generalizados en los países de Europa pudiéndose encontrar prácticamente los mismos. El juego del marro no era una excepción. Universalmente conocido ha perdurado vivo hasta no hace mucho. Que la popularidad del juego se prolongara hasta principios del siglo XX dice mucho de la importancia que siempre tuvo desde su ancestral origen. Uno de los autores de este artículo, recuerda haberlo jugado con mucha entrega y pasión en sus tiempos de infancia. Asimismo hemos comprobado en relatos de familiares nacidos a inicios del s. XX que el juego era muy presente en sus vidas y se practicaba en cualquier terreno y en numerosas ocasiones. Hoy prácticamente podemos sentenciar que el juego del marro ha perecido definitivamente al paso del tiempo. Durante siglos ha sido la delicia de los jóvenes y sirvió de recurso para una educación física natural, espontánea y libre, que endurecía el cuerpo y desarrollaba el carácter.

Por mucho que nos esforcemos, los juegos tradicionales han perdido espacio y dimensión en el currículo de la educación Primaria y Secundaria. El grado de marginalidad de algunos de estos juegos puede ser reparado como un excelente contenido para el desarrollo competencial e interdisciplinar. El marro puede ser un juego recuperado y a través de él, tal y como ya hiciera Pere Vergés, podemos enlazar contenidos del área de Educación Física con otras competencias educativas básicas, una de ellas puede ser la de explicar el valor social de su historia. Pero también debemos revalorizar su recuperación por la calidad sociomotriz que implica y el enorme beneficio que concede al desarrollo de las capacidades condicionales y cognitivas.

Prisionero del tiempo, hoy hemos rescatado la esencia humana del juego del marro, y convencidos de que nunca volverá a ser lo que fue, podemos restablecer su memoria e incluso suscitar el renacimiento eventual o temporal de su práctica.

Siguiendo a Vicente y Brozas (1996) el dilema que se nos platea no es fácil. Como otros juegos de su casta en la tradición de la cultura no dominante, el marro se presenta como valor etnológico y crítico ante la tradición (es)forzada del deporte. Aquí yace el valor que supone preservar o conservar la tradición, pero también el de 
proceder a los valores de la tradición renovados, apuntando a una posible didáctica crítica en donde la deportivización del juego sea presentada como alternativa a los deportes dominantes.

\section{Referencias bibliográficas}

ALCOCER, F. de (1559). Tratado del juego. Salamanca: Andreas de Portonariis.

ÁLVAREZ, E. (1995). La actividad físico-lúdica en el siglo de Oro español. Apunts. Educación Física y Deportes, 42, 7-25.

AMAR, A. y JAUFFRET, L. F. (1807). La gimnástica o escuela de la juventud, tratado elemental de juegos, de ejercicios considerados en razón de su utilidad física y moral. Madrid: Imp. Álvarez.

ANDREU, E. (2009). El juego infantil mediterráneo: Grecia antigua. Aloma, 25, 39-51.

AUTORIDADES [Real Academia Española] (1734). Diccionario de la lengua castellana, t. IV. Madrid: Imp. de la Real Academia Española, Herederos de Francisco Hierro.

BANTULÀ, J. (2005). El joc popular i tradicional als segles XVI i XVII: revisió des d’una perspectiva taxonómica. Educació i Història, 8, 134-146.

BANTULÀ, J. (2006). La introducció dels jocs populars i tradicionals en l'educació escolar dels infants. Temps d'Educació, 8, 235-247.

BANTULÀ, J. (2008). Per una pedagogia del joc: la seva aposta educativa des del segle XIX. Revista Catalana de Pedagogia, 6, 361-380.

BANTULÀ, J. y VILANOU, C. (2009). Joc, humanisme i pedagogia: la virtut de l'eutrapèlia. Aloma, 25, 53-89.

BARRILERO, P. (1900). Juegos gimnásticos. Cuaderno núm. 1 El Cricket. Madrid: Imp. de los Hijos de G. M. Hernández.

BARTRÉS, R. (1934). Cròniques. Garbí, 6, 6.

BECERRO-MINA, A. y TORREBADELLA-FLIX, X. (2013). El joc tradicional i popular a l'escola primària: una proposta de l'educació física cap a la cessió d'autonomia. REIRE: Revista d'Innovació i Recerca en Educació, 6(2), 100-118.

BORJA, M. de (1982). El joc, eina pedagògica a Catalunya. Barcelona: La llar del llibre.

BRASÓ, J. y TORREBADELLA, X. (2014). El joc del rescat a Catalunya. Un projecte educatiu a l'Escola del Mar de Pere Vergés. Temps d'Educació, 46, 191-212.

CARO, R. (1978). Días geniales o lúdicros. Madrid: Espasa Calpe. [Edición, estudio preliminar y notas de Jean-Pierre Etienvre]

CASALS, M. (ca. 1930). Gimnasia, juegos y deportes. Manual de educación física recreativa. Barcelona: Casals. 
CASTELLANOS, B. S. (1849). Costumbres españolas. De los juegos infantiles. Museo de los niños, 3, 295-297.

CASTRO, N. (1895a). Educación física de las niñas. La Escuela Moderna, 50, 362.

CASTRO, N. (1895b). Educación física de las niñas. La Escuela Moderna, 49, 288298.

COBARRUVIAS, P. (1543). El Remedio de Jugadores. [Salamanca]: Juan de Juntas.

COBARRUVIAS, S. de (1611). Tesoro de la lengua castellana o española. Madrid: Imp. Luis Sánchez.

CONDO, A. (1927). Gimnasia e hidroterapia. Madrid: Suc. Rivadeneyra.

CORMON, F. (1776). Sobrino aumentado o Nuevo diccionario de las lenguas española, francesa y latina, t. II. Amberes: Hermanos de Tournes.

DAVIU, M. (1902). Juegos corporales VIII. El Magisterio Balear, 5, 41-42.

DEVIS, J. (2008). El juego en el currículum de la Educación Física: aproximación crítica (o el reino de lo posible en la postmodernidad). Revista de Educación Física y Deporte, 27(2), 79-89.

DIEM, C. (1966). Historia de los deportes, vol. II. Barcelona: Luis de Caralt.

ELEIZEGUI, J. de (1924). Los juegos en la infancia. Barcelona: Sociedad General de Publicaciones.

ELIAS, N. y DUNNING, E. (1992). Deporte y ocio en el proceso de civilización. Madrid: FCE.

ELSCHENBROICH, D. (1979). El juego de los niños. Estudios sobre la génesis de la infancia. Madrid: Zorro.

ESCRIBANO, G. (1912). Ejercicios corporales. Madrid: Imp. de la Enseñanza.

FARRÉ, J. (2009). Diari dels últims mesos de Guerra Civil a Torredembarra. Recull de Treballs, 10, 5-31.

FEDERACIÓN GIMNÁSTICA ESPAÑOLA (1902). Ferias y Fiestas populares de Ntra. Sra. de las Mercedes. Programa y Reglamentos de los concursos de la Federación Gimnástica Española. Barcelona: Imp. José Cunill.

FERNÁNDEZ, Á. (1852). Los juegos en las diferentes edades en todos los pueblos del mundo desde la antigüedad más remota hasta nuestros días. Madrid: Biblioteca Universal.

FERNÁNDEZ, F. (1864). Juegos y entretenimientos de las niñas. Madrid: Imp. del Est. de Mellado.

FERNÁNDEZ, J. (1898, 8 de febrero). La Ilustración Española y Americana, 70.

FERNÁNDEZ, J. (1902, 8 de mayo). Crónica general. La Ilustración Española y Americana, 266. 
FERRER, D. (1893). Programa de lecciones de gimnasia explicadas por el profesor de esta asignatura. Barcelona: Imp. J. Farriols y Amal.

FERRER, D. (1900a, 30 de diciembre). Cartilla de gimnasia higiénica. Los Deportes, 52, 820-822.

FERRER, D. (1900b, 10 de junio). Lo que deben ser los juegos en los niños. Los Deportes, 23, 355-356.

FERRER, D. (1901). Cartilla gimnástica higiénica de carácter popular para la divulgación y propaganda de esta índole de conocimientos. Barcelona: L'Avenç.

FERRER, E. (1919). Resumen de las lecciones teóricas y prácticas de gimnástica (7 ed.). Palma de Mallorca: Est. Tip. de Francisco Soler.

FONSSAGRIVES, J. B. (1877). Educación física de las niñas. Ejercicios físicos y juegos IV. La Instrucción Pública, 58, 131-133.

Gálvez, G. (1940). Pedagogía Española. Antología del Padre Manjón. Madrid: Editorial Magisterio Español.

GARCÍA, P. de A. (1882). Teoría y práctica de la educación y la enseñanza. De la educación física, t. V, $1^{\text {a }}$ parte. Madrid: Gras y $\mathrm{Ci}^{\mathrm{a}}$ Editores.

GARCÍA, P. de A. (1896). Compendio de pedagogía teórico-práctica. Madrid: Lib. Viuda de Hernando y $\mathrm{C}^{\mathrm{a}}$.

GARCÍA-FRAGUAS, J. E. (1896). Tratado racional de gimnástica y de los ejercicios y juegos corporales..., t. II Teoría general de la gimnástica y del Sport. Madrid: Casa editorial y Lib. de la Viuda de Hernando y $\mathrm{Ci}^{\mathrm{a}}$.

GARCÍA-FRAGUAS, J. E. (1897). Programa ilustrado de advertencias y figuras de actitudes para las practicas de Gimnasia Higiénica-Sports, Juegos y ejercicios medicinales en los institutos, colegios. Valencia: Imp. Ripollés.

GINER, F. (1887). El ayuntamiento de Madrid y el juego de los niños. BILE, 259, 338339.

GONZÁLEZ, J. A. (1993). Tractatus ludorum: Una antropología del juego. Barcelona: Anthropos.

GUIBERT, M. E. (1990). La renovación pedagógica de los maestros de Tudela a finales del siglo XIX: paseos escolares y enseñanza de la Geografía. Revista del Centro de Estudios Merindad de Tudela, 2, 25-50.

GUILLEMARD, G.; MARCHAL, J. C.; PARENT, M.; PARLEBAS, P. y SCHMITT, A. (1988). Las cuatro esquinas de los juegos. Lérida: Agonos.

GUIMERÁ, I. (1889). Los ejercicios corporales. BILE, 288, 33-37.

HARDY, T. (2011). Lejos del mundanal ruido. Barcelona: Alba Editorial.

HERNÁNDEZ, J. L. y Caballero J. (ca, 1928). Manual de educación física elemental. Barcelona: Núñez y Ca, S en C. 
HERNÁNDEZ, M. (2004). Estudio antropológico del juego deportivo en España desde sus primeras ilustraciones gráficas hasta la edad moderna. Madrid: Facultad de Ciencias de la Actividad Física y el Deporte de la Universidad Politécnica de Madrid. [tesis doctoral]

HERNÁNDEZ, S. (ca. 1900). Juegos de los niños en las escuelas y colegios. Madrid: Saturnino Calleja Fernández.

HESSE, J. (1967). El deporte en el siglo de oro. Madrid: Taurus.

Higiene (1905, 10 de noviembre). Ciudad Lineal, 3.

HUGHES, T. (1923). Tomás Brown en la escuela, t. I. Madrid: Calpe.

HUIZINGA, J. (1957). Homo Ludens. Buenos Aires: Enece.

J. L. N. P. R. (1808). Sobre la gimnástica de la juventud, y si conviene que los niños aprendan a jugar científicamente. Minerva, 25, 25-32.

JULLIEN, M. A. (1845). Ensayo General de educación física, moral e intelectual (2a ed.). Valencia: Imp. José Mateu Cervera.

KANT, I. (2003). Pedagogía. Madrid: Akal.

LAGARDERA, F. y LAVEGA, P. (2003). Introducción a la praxiología motriz. Barcelona: Paidotribo.

LAVEGA, P. (2006). El juego y la tradición en la educación de valores. Educación Social, 33, 54-72.

LEDESMA, A. (1611). Juegos de nochebuena moralizados a la vida de Christo, martirio de Santo y reformación de costumbres. Madrid: Alonso Martín.

LÓPEZ, F. (1855). Recreo de la infancia. Colección de juegos para niños de ambos sexos. Madrid: Imp. de D. Antonio Pérez Dubrull.

LÓPEZ, F. (1998a). Historia de la educación física de 1876 a 1898. La Institución Libre de Enseñanza. Madrid: Ed. Gymnos.

LÓPEZ, F. (1998b). Los juegos en la Institución Libre de Enseñanza. Ensayos, 13, 249-264.

MACORRA, F. de la (1894). Programa de la asignatura de gimnástica higiénica y juegos corporales. Madrid: Tip. M. G. Hernández.

MANCUVRIER, E. (1890). El concurso de Bischoffsheim sobre la organización de los juegos escolares. BILE, 319, 150-154.

MARCO, L. y OCHOA, E. de (1896). Repertorio completo de todos los juegos. Madrid: Lib. Ed. de Bailly-Bailliere e Hijos.

Marín, E. (2009). D. Marcelo Santos Sanz Romo, iniciador y propagandista de la educación física española: vida y obra. Universidad de Alcalá, Departamento de Didáctica. [Tesis doctoral, disponible en: http://dspace.uah.es/dspace/handle/10017/6344] 
MASPONS, F. (1874). Jochs de la infancia. Barcelona: Estampa de Frederich Martí y Cantó.

MAYOR, A. (2002). Historia de la educación física infantil en España en el siglo XIX. Alcalá de Henares: Servicio de Publicaciones de la Universidad de Alcalá.

MEDINA, X. y Sánchez, R. (2003). Culturas en el juego: Ensayos de Antropología del deporte en España. Barcelona: Icaria.

MÉNDEZ, F. (1907). Robo de las Lilas. Por esos mundos, 157, 190-192.

MERCURIAL, J. (1845). Arte jimástico-médico. Madrid: Imp. Victoriano Hernando.

MINISTERIO DE LA GUERRA (1911). Reglamento Provisional de Gimnasia para Infantería. Madrid: Talleres del Depósito de la Guerra.

MUTEL, M. (1846). Higiene militar, t. II. Madrid: Lucas González y Cia

NAVARRO, V. y TRIGUEROS, C. (2008). Líneas de investigación del juego motor y modelos de estudio en el ámbito de la Educación Física (1990-2007). Revista Iberoamericana de Educación, 46, 1-12.

Nociones de ejercicios corporales, 1r curso (1909). Málaga: Zambrano Hermanos.

OSSORIO, M. (1891). Gente menuda (Romances infantiles). Madrid: Imp. Manuel Minuesa de los Ríos.

PALACIO, E. de (1882, 15 de septiembre). Inclinaciones. La Ilustración Española y Americana, 158.

PARLEBAS, P. (2001). Juegos, deporte y sociedad. Léxico Praxiología motriz. Barcelona: Paidotribo.

PAVET, C. (1827). Higiène des colléges et des maisons d'éducatión des deux sexes. Paris: Jobard.

PAYÀ, A. (2004). Joc corporal, esport i educació física a l’ideari pedagògic de la Institución Libre de Enseñanza. Educació i Història, 7, 117-133.

PAYÀ, A. (2006). La actividad lúdica en la historia de la educación española contemporánea. Departamento de Estudios Sociales de la Universidad de Valencia [tesis doctoral, disponible en: http://www.tdx.cat/handle/10803/9701]

PAYÀ, A. (2007). Consideraciones pedagógicas sobre los valores y posibilidades educativas del juego en la España contemporánea (1876-1936). Revista Historia de la Educación, 26, 299-325.

PELEGRÍN, A. (1998). Repertorio de antiguos juegos infantiles. Madrid: CSIC.

PELEGRíN, A. (2000). Juegos de aldeas y de la niñez en textos hispanos (1510-1820). En P. Irureta-Goyena (Coord.). El juego en el libro antiguo (pp. 23-52). Madrid: Universidad Politécnica de Madrid-INEF.

PUSALGAS, I. (1831). Manual de higiene arreglado según la doctrina de sir John Sinclair. Barcelona: J. Rubio. 
QUEVEDO, F. J. (1992). El cuento como atracción a la lectura: "Patio de Armas" de Ignacio Aldecoa. El Guiniguada, 3(1), 167-174.

R. A. E. (1803). Diccionario de la lengua castellana. Madrid: Viuda de Ibarra.

R. A. E. (1852). Diccionario de la lengua castellana. Madrid: Imp. Nacional.

R. C. (1847). Juegos de jardín o campo. Madrid: Imp. R. Fonseca.

Remón, A. (1623). Entretenimientos y juegos honestos y recreaciones cristianas. Madrid: Viuda de Alonso Martín a costa de Lucas Ramírez.

RUBIO, R. (1889). El movimiento pedagógico francés en 1888. BILE, 289, 49-54

RUBIO, R. (1893). Los juegos corporales en la educación. BILE, 391, 145-150.

RUMREP, J. (1903, 29 de mayo). El Colegio de María Cristina. La Correspondencia Militar, 1-2.

SAN MARTÍN, A. (1889). De los juegos corporales más convenientes en España. El Ateneo-revista científica, literaria y artística, 3, 53-75.

SANCHA, J. (1855). Biblioteca de Autores Españoles... Romancero y Cancionero Sagrados. Madrid: Imp. de Rivadeneyra.

SANZ, M. (1894). Programa de gimnástica higiénica y juegos corporales. Madrid: Imp. J. Fernández.

SEJOURNANT, M. de (1759). Nouveau dictionnaire espagnol-François et latin, t. I. Paris: Charles-Amtoine Jombert.

SIMON (1827). Traité d'hygiène appliquée à l'éducation de la jeunesse. Paris: Villeret.

SIMÓN, J. (1992). Historia del Colegio Imperial de Madrid (1346-1955). Madrid: Instituto de Estudios Madrileños.

SUÁREZ, C. (1630). Plaza universal de todos ciencias y artes. Perpiñan: Luis Roure.

Temas navales. Las últimas maniobras de nuestra escuadra (1932, 4 de julio). Luz, 11.

TISSIÉ, Ph. (1899). La fatiga. El adiestramiento físico. Madrid: Lib. de Fernando Fé.

TORREBADELLA, X. (2009). Contribución a la historia de la educación física en España. Estudio bio-bibliográfico en torno a la educación física y el deporte (18001939). Lleida: Universitat de Lleida Departament d'Història de l'Art i Història Social [tesis doctoral no publicada].

TORREBADELLA, X. (2011). Repertorio bibliográfico inédito de la educación física y el deporte en España (1800-1939). Madrid: Fundación Universitaria Española.

TORREBADELLA, X. (2012a). Las primeras revistas profesionales y científicas de la educación física española, 1882-1936. Apunts. Educación Física y Deportes, 109, 11-25. doi: 10.5672/apunts.2014-0983.es.(2012/13).109-01 
TORREBADELLA, X. (2012b). Orígenes del fútbol en Barcelona (1892-1903). Revista Internacional de Ciencias del Deporte, 27, 80-102. doi:10.5232/ricyde2012.02706

TORREBADELLA, X. (2013). Gimnástica y educación física en la sociedad española de la primera mitad del siglo XIX. Lleida: Universitat de Lleida.

TUDELA (1835). Ordenanzas municipales de la ciudad de Tudela. Pamplona: Imp. Francisco Erasun.

UN AFICIONADO (ca. 1905). Manual de juegos y deportes. Madrid: Biblioteca popular Saturnino Calleja.

UN PAPA (1897). Los juegos de la infancia. París: Garnier Hermanos.

VALLÈS, J. (1889). Jacques Vingtras. L'enfant. Paris: Charpertier.

VÁZQUEZ, F. y SEOANE, J. B. (2004). España y la cruzada médica contra la masturbación (1800-1900). Elementos para una genealogía. Hispania, 218, 835868.

VICENTE, M. y BROZAS, M. P. (1996). La disposición regulada de los cuerpos. Propuesta de un debat sobre la cultura física popular y los juegos tradicionales. Apunts. Educación Física y Deportes, 48, 6-16.

\section{Correspondencia con los autores}

Xavier TORREBADELLA FLIX

Facultad de Ciencias de la Educación

Universidad Autónoma de Barcelona

Campus de la UAB - Edificio G6, despacho 168

08193- Bellaterra (Cerdanyola del Vallès). ESPAÑA

e-mail: franciscoxavier.torrebadella@uab.cat

Teléfono: 93-5812671 